\title{
Superheavy-element spectroscopy: Correlations along element 115 decay chains
}

D. Rudolph, U. Forsberg, L.G. Sarmiento, P. Golubev, and C. FAHLANDER

Department of Physics, Lund University, 22100 Lund, Sweden

\begin{abstract}
Following a brief summary of the region of the heaviest atomic nuclei yet created in the laboratory, data on more than hundred $\alpha$-decay chains associated with the production of element 115 are combined to investigate time and energy correlations along the observed decay chains. Several of these are analysed using a new method for statistical assessments of lifetimes in sets of decay chains.
\end{abstract}

\section{Introduction}

"The quest for superheavy elements is driven by the desire to find and explore one of the extreme limits of existence of matter" [1]. It relates to fundamental questions concerning the properties of the strong nuclear force capable of forming the heaviest atomic nuclei yet known. The question of whether superheavy elements can be formed in stellar processes is also of fundamental nature. In chemistry superheavy elements pose questions such as: What are their properties? How do they fit into the existing scheme of the periodic table? In fact, the international superheavy-element community will discuss their past, presence and future in the framework of an approved Nobel Symposium on Chemistry and Physics of Heavy and Superheavy Elements near Lund in Spring 2016. 
"More than 40 years ago, an island of stability of superheavy nuclei was predicted by nuclear structure theorists (see, e.g., Ref. [2]). This island rests on increased nuclear stability due to anticipated magic proton and neutron numbers at spherical nuclear shape (see, e.g., Ref. [3]), while surrounding archipelagos may also arise from increased nuclear binding of deformed atomic nuclei $[4,5]$. Even today theoreticians face the challenge or have the freedom - to extrapolate over several tens of mass units towards the island, based on quite reliable spectroscopic information in the region around nobelium and rutherfordium [6].

Along their way, experimentalists have not only been producing heavier and heavier atomic nuclei, but also added more and more chemical elements to the Periodic Table. Present-day research builds upon one-atom-at-a-time creation and observation. In this context, 'time' refers to typically a day, a week, or as much as several months - and it is at this tiny rate that detailed spectroscopic information needs to be obtained.

During the past 15 years, correlated $\alpha$-decay chains have been observed in a number of experiments performed at the Flerov Laboratory of Nuclear Reactions (FLNR) in Dubna, Russia. Beams of ${ }^{48} \mathrm{Ca}(Z=20)$ impinged on actinide targets $(Z=92-98)$. The decay chains were interpreted to start from isotopes of elements $Z=112-118$, produced via the mechanism of fusion-evaporation reactions $[7,8]$. The $\alpha$-decays of nuclei with even proton number, $Z$, and even mass number, $A$, typically proceed from ground-state to ground-state. The decay energies along such decay chains are thus characteristic for a given decay step and can be used as a fingerprint for a given isotope of a certain element [9].

Decay patterns are usually much more complex for $\alpha$ decays of odd- $A$ or especially odd-odd nuclei. Their $\alpha$ decays usually proceed to excited states in the daughter nucleus [10]. On the one hand, decay into excited states hampers indirect methods of determining $Z$, not least because decay chains along the same isotopes may follow different decay paths depending on the starting point of a certain isotopic decay sequence. On the other hand, the possibility arises to observe $X$-ray photons in the course of electromagnetic internal conversion decays of the excited states in $\alpha$-decay daughter nuclei [11]. $X$-ray energies are predicted with high precision even for the heaviest elements since long (see, e.g., Ref. [12]), and they are characteristic for a given proton, hence atomic, number [13]. In addition, high-resolution $\alpha$-photon coincidence spectroscopy - such as pioneered on element $Z=115$ decay chains - closes in towards nuclear structure studies near the island of stability [14]" [15]. 


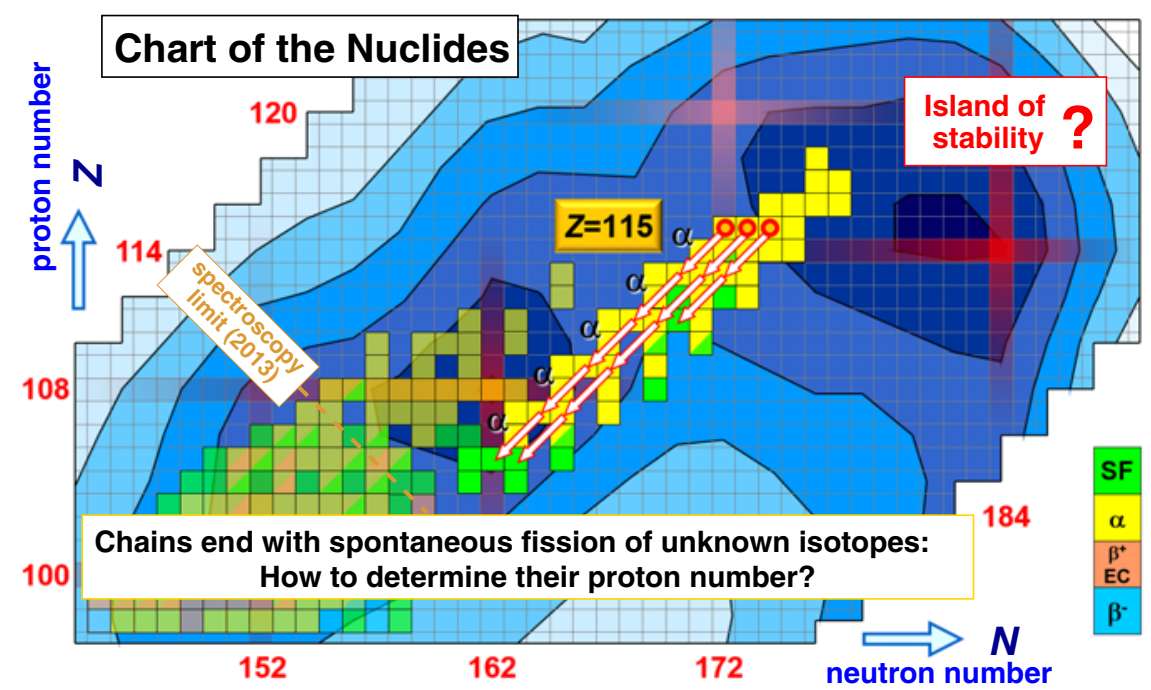

Figure 1: Upper end of the chart of atomic nuclei. Each square in this twodimensional chart represents a certain isotope comprising $Z$ protons and $N$ neutrons. Red lines denote possible 'magic numbers'. The combination of $Z=114$ and $N=184$ is one of several long-standing predictions for the centre of the island of stability [2]. Bright shading indicates observed isotopes, with $\alpha$-decay chains of element 115 indicated by diagonal arrows. The darker the blue background, the more stable the isotopes are predicted to be. A coarse '2013 $\alpha$-photon spectroscopy limit' is indicated by a dashed orange line.

The status of the field is coarsely illustrated in fig. 1, and detailed from the point of view of the TASCA collaboration ${ }^{1}$ in, for instance, Refs. $[16,17]$. Independent confirmation of the production of elements up to $Z=117$ has been achieved [18], months-long search experiments for elements 119 and 120 conducted, and gas-phase chemistry of flerovium, $Z=114$, being tackled [19]. The subject of this article is an extended analysis of correlations of decay times and decay energies along element 115 decay chains.

\section{Correlation analysis}

Until now, experimental data on in total $113 \alpha$-decay chains associated with the direct production of isotopes of element 115 (placeholder name ununpentium, Uup) have been published [14,20-23]. Following the first observation of four decay chains of element 115 at the Dubna gas-filled

\footnotetext{
1 "TransActinide Separator and Chemistry Apparatus" at GSI Helmholtzzentrum für Schwerionenforschung, Darmstadt, Germany, see also https://www-windows.gsi.de/tasca/
} 
recoil separator (DGFRS) [20], decay energies and correlation times from 37 element 115 "Dubna chains" are summarized in tables II, III, and IV of ref. [21]. More recently, high-resolution $\alpha$-photon coincidence data taken at the TASCA facility at GSI Darmstadt resulted in 30 element 115 "TASCA chains" (cf. supplemental material of ref. [14] and table I of ref. [22]), while a similar experiment conducted at the Berkeley gas-filled separator gave rise to additional 46 element 115 "Berkeley chains" (cf. supplemental material of ref. [23]) - and $37+30+46=113$. The most relevant observables are decay energies, $E_{i}$, for a given decay step $i$ along an $\alpha$-decay chain, and correlation times between

- the implantation of a recoiling element 115 nucleus into a position sensitive silicon-detector arrangement and the first $\alpha$ decay, $\Delta t_{1}$,

- between $n$ subsequent $\alpha$ decays, $\Delta t_{i}, i=2, \ldots, n$,

- and between the last correlated $\alpha$ decay and a spontanous fission (SF) event terminating the chain after $n \alpha$-decay steps, $\Delta t_{n+1}$.

Note that decay energies, $E_{i}$, can involve summing related to $\alpha$-particle emission and subsequent prompt emission of conversion electrons in case $\alpha$ decay feeds excited states in the daughter nucleus, which can quickly decay into lower-lying states by electromagnetic decay processes [24].

Out of these in total 113 decay chains of directly produced evaporation residues (ER) of element $115,4+7+3=14$ chains are of type ER- $\alpha(-$ $\alpha)$-SF $(n=1,2)$, while the remaining $33+23+43=99$ chains comprise five subsequent $\alpha$ decays $(n=5)$, before SF events conclude the chains after typically several hours $[14,21,23]$. Note that it until now has not been possible to experimentally decide whether or not these SF events are preceded by an electron capture (EC) decay.

Inspecting the correlation times and decay energies observed for each individual decay step and eventually applying statistical measures such as the "Schmidt test" [25] yields that these $99 n=5$ chains are consistent with two different decay sequences. See, for instance, ref. [15] for the latest numerical compilation. Folding the mean excitation energy of the compound nucleus ${ }^{291} 115$ into the picture, $2+1+0=3$ decay chains are associated with the production of ${ }^{287} 115$, i.e. the 4 -neutron $(4 n)$ evaporation channel, while the vast majority of $31+22+43=96$ chains are considered to represent the production of ${ }^{288} 115$ in the $3 n$-evaporation channel.

Given that ${ }^{288} 115$ is an odd-odd nucleus, it is noteworthy that the description of such a comprehensive ensemble of 96 long $\alpha$-decay chains is consistent with the observation of just one decay sequence. In turn, more detailed inspections of partly broad distributions of decay energies and, for 

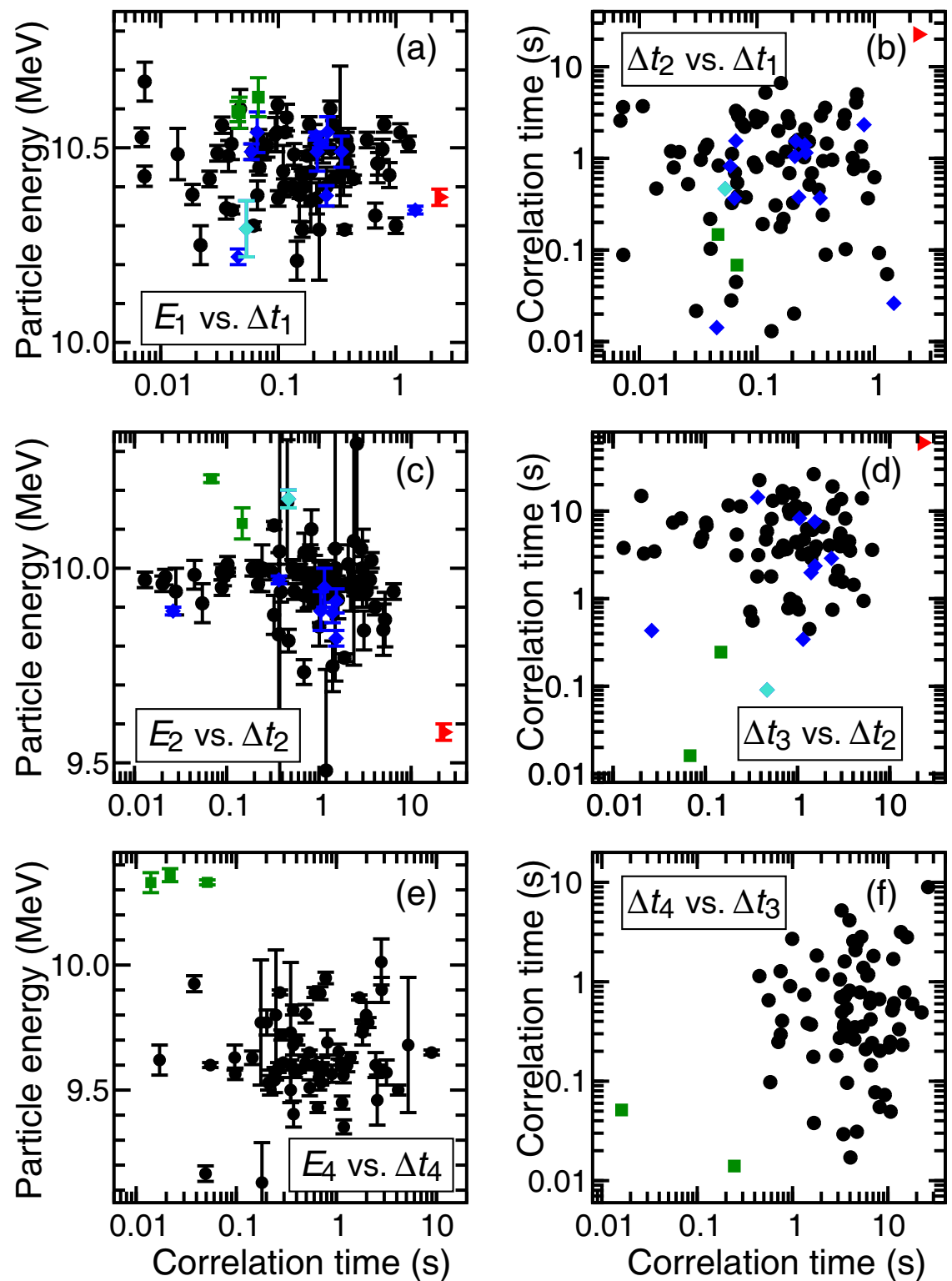

Figure 2: Two-dimensional histograms of measured decay energies and correlation times of decay step 1 [panel (a)], decay step 2 [panel (c)], and decay step 4 [panel (e)] of in total $113 \alpha$-decay chains associated with isotopes of element $115[14,21$ 23]. Filled black circles correspond to data from 96 five- $\alpha$-decay long chains of ${ }^{288} 115$ and filled green squares to data from 3 five- $\alpha$-decay long chains of ${ }^{287} 115$. Filled blue diamonds represent data from 12 out of 14 recoil- $\alpha(-\alpha)$-fission chains. The chains denoted D3 and D4 (cf. table III in ref. [21]) are emphasized as red triangles and turquoise diamonds, respectively. Panels (b),(d),(f) illustrate relations of correlation times of subsequent decay steps along element 115 chains. 
the first time in this superheavy regime, $\alpha$-photon coincidence spectroscopy, give rise to first glimpses of solid experimental information for detailed nuclear structure assessments of the heaviest atomic nuclei. This implies decay schemes including excited states, $\gamma$-ray decays, and $\alpha$-decay hindrance factors, to name but a few $[14,15,23,26]$.

Prior to more comprehensive statistical assessments of decay chains as a whole (cf. next section and ref. [22]), relations between correlation times and decay energies of a given decay step or two correlation times of, for instance, subsequent decay steps can be used to identify candidates for different ensembles of decay chains. The six panels shown in fig. 2 are examples of such a graphical analysis.

The data points of the 96 chains associated with ${ }^{288} 115$, displayed as filled black circles in fig. 2, can be considered to form a reference for any of the other element 115 decay chains. For instance, due to the broad range of decay energies and similar correlation times - and, thus, half-lives, $T_{1 / 2}$ $[14,15,21,23$ ] - data for decay step 1 [panels (a) and (b)] does not allow to distuingish the three chains associated with ${ }^{287} 115$, displayed as filled green squares, from the ${ }^{288} 115$ reference. However, a clear trend becomes visible in decay step 2 [panels (c) and (d)] due to somewhat higher decay energies as well as shorter correlation times. Finally, data associated with decay step 4 [panels (e) and (f)] allow for an apparent visual distinction, which can also be turned into a significant statistical distinction due to significantly higher decay energies and shorter correlation times. The statement is backed up by the fact that the three ${ }^{287} 115$ chains are observed at higher excitation energies of the compound nucleus ${ }^{291} 115$, as mentioned earlier [14,21].

While this situation for the long $n=5$ element 115 decay chains is rather straightforward and undisputed, the interpretation of the remaining 14 "short chains" $(0+2+2=4$ ER- $\alpha$-SF, $n=1$, and $4+5+1=10$ ER- $\alpha$ $\alpha$-SF, $n=2$ ) is less clear. Twelve of these chains are shown in fig. 2 as blue diamonds, while the chains denoted D3 and D4 (cf. table III in ref. [21]) are emphasized as red triangles and turquoise diamonds, respectively.

Looking at the relevant panels (a)-(d) of fig. 2 and comparing the distributions of the black circles and blue diamonds, there is no apparent difference. In fact, this correspondence also holds numerically [15,22]. Thus, for any of these twelve short chains it is impossible to decide whether it represents a possible EC or SF branch in either ${ }^{284} 113$ or ${ }^{280} \mathrm{Rg}$ [17], or whether it belongs to an independent sequence starting from ${ }^{289} 115$, i.e. the isotope representing the $2 n$ evaporation channel of the reaction ${ }^{48} \mathrm{Ca}+{ }^{243} \mathrm{Am}$. Of course, such an $\alpha$-decay sequence would have to have very similar decay characteristics as the first three decay steps of the long ${ }^{288} 115$ reference. 
In fact, arguments based on reaction cross sections and compound nucleus excitation energies suggest that these short chains are shared about 50:50 between ${ }^{288} 115$ and ${ }^{289} 115$ [22].

Two short chains deserve special attention. Chain D4 [21], represented by turquoise diamonds in fig. 2, is indistinguishable from the black circles and blue diamonds as far as decay step 1 is concerned [panels (a) and (b)]. However, the energy of decay step 2 [panel (c)] as well as the decay-time correlation between steps 2 and 3 [panel (d)] places this chain in the vicinity of the three long decay chains associated with ${ }^{287} 115$ (green squares). A definite assignment to any of the isotopes of element 115 is not possible though. Finally, the decay characteristics reported for chain D3 [21], represented by red triangles in fig. 2(a)-(d), suggest that it has a different origin than any other of the 113 chains associated with element 115. The red triangles show up in the upper right corner of panels (b) and (d) and in the lower right corner of panel (c), This is due to three exceptionally long correlation times and one exceptionally low decay energy, i.e. due to four unusual out of five possible parameters, clearly indicating that this chain is distinct. It may form a second, independent decay sequence of ${ }^{289} 115$ [17, 22].

\section{Statistical assessments}

An alternative approach to assess the chains is the relatively "new test" suggested by Schmidt [25]. Here, the standard deviation of the logarithms of measured lifetimes $\theta_{i}=\ln \left(t_{i}\right)$ in a set of $m$ decays of one decay step in a given decay chain is compared with expected values thereof. Too large standard deviations indicate that the set of decays might not originate from one single radioactive species, and too small values indicate a lack of sensitivity in the experiment, or that the data set is unjustly pruned. The fundamental principle behind this test is the fixed shape of a probability density function (PDF) on a logarithmic scale for an exponential decay, ensuring that the expected standard deviation is independent from the half-life. When the number $m$ of decays is large, the expectation value of the standard deviation is 1.28 , and is found in the interval $1.28 \pm 2.15 / \sqrt{m}$ with $90 \%$ probability. For small values of $m$, the upper and lower limits are tabulated in ref. [25].

Applied to the short chains, the standard deviations are 1.20, 1.75, and, 1.84 for the first, second and third decay step, respectively. For steps one and two the number of chains is $m=14$, and for the third step $m=10$. Comparing with the $90 \%$ confidence intervals, $[0.73,1.77]$, [0.73,1.77] and $[0.65,1.82]$, respectively, the assignment to only one radioactive decay species is seemingly justified. 
However, this measure only applies to one decay step at the time. Hence, not all of the available information is considered. For example, a data set containing one exceptionally long correlation time in each step can be correctly judged sound using the test, while there might still be reasons to question the assignment if the long correlation times all come from the very same chain. Therefore, we propose a generalisation of the test.

Assume that $m$ chains have been observed and that each chain $i$ contains $n$ decay steps. Let $\theta_{i_{j}}$ be the logarithm of the $j$ :th lifetime in chain $i$. Then, the measure

$$
\xi_{m, n}=\sqrt[2]{\frac{\sum_{i=1}^{m} \sqrt[n]{\Pi_{j=1}^{n}\left(\theta_{i_{j}}-\bar{\theta}_{j}\right)^{2}}}{m}}, \bar{\theta}_{j}=\frac{\sum_{i=1}^{m} \theta_{i_{j}}}{m}
$$

will take into account correlation times observed along decay chains rather than in single decay steps. By multiplying the deviations $\left(\theta_{i_{j}}-\bar{\theta}_{j}\right)^{2}$ with each other, all data on correlation times from one chain are considered jointly. Introducing the roots of order $n$ allows for a further generalisation to be introduced later.

Figure 3 shows simulated distributions of $\xi_{m, n}$ for $m=14$ and $n=1,2$ and 3. Two million sets of correlation times have been randomly drawn from exponential PDFs. This measure, just like the one proposed by Schmidt, is independent from the half-life. When more decay steps $n$ are considered, the distributions of $\xi_{m, n}$ get more narrow. Varying the number of chains $m$ modifies the shape as well, with larger sets of chains leading to more narrow distributions. For each $m$ and $n$ the expectation value and the $90 \%$ confidence intervals can be tabulated. Some values are given in the left part of table 1.

Applying this generalised test to the short $n=1,2$ chains associated with element 115 is unfortunately not straight forward due to the fact that the chains are of different length. This motivates a further generalisation of eq. 1 , where the number of decays in chain $i$ is $n_{i}$ :

$$
\xi_{m, n_{i}}=\sqrt[2]{\frac{\sum_{i=1}^{m} \sqrt[n_{i}]{\Pi_{j=1}^{n_{i}}\left(\theta_{i_{j}}-\bar{\theta}_{j}\right)^{2}}}{m}}
$$

The averages $\theta_{i_{j}}$ are calculated using the respective numbers of available correlation times in that decay step. To construct relevant confidence intervals, the structure of each chain, i.e., its length and the number and position of potentially missing members need to be taken into account in the simulation. For the purpose of evaluating the short chains, it is sufficient to 
Table 1: Left: Expectation values $\langle\xi\rangle, 90 \%$ confidence intervals (5\% of the probability density lies below " $5 \% \downarrow$ " and $5 \%$ lies above " $\uparrow ")$ of $\xi$ for various values of $m, n$, and $l$. The upper left part of the table relates to $\xi_{m, n}$, i.e. sets where all chains have equally many decay steps. The lower left part refers to $\xi_{m, n, l}$, where $l$ chains end earlier. The right part presents $\xi_{m, n_{i}}$ values when the respective chain is excluded from the data set. Those marked with * should be compared with $\xi_{m=13, n=3, l=3}$, and the others with $\xi_{m=13, n=3, l=4}$. See text for details.

\begin{tabular}{llllll||ll|ll}
$m$ & $n$ & $l$ & $<\xi>$ & $5 \% \downarrow$ & $5 \% \uparrow$ & Chain & $\xi_{m, n_{i}}$ & Chain & $\xi_{m, n_{i}}$ \\
\hline 14 & 2 & - & 0.93 & 0.65 & 1.27 & D1 & 1.34 & C4 & 1.22 \\
13 & 2 & - & 0.93 & 0.64 & 1.28 & D2 & 1.34 & C5 & 1.35 \\
10 & 3 & - & 0.82 & 0.57 & 1.12 & D3 & 1.03 & C6 & 1.35 \\
9 & 3 & - & 0.81 & 0.55 & 1.13 & D4 & 1.33 & C7 & 1.34 \\
14 & 3 & 4 & 0.86 & 0.62 & 1.15 & C1 & $1.36^{*}$ & B1 & 1.35 \\
13 & 3 & 4 & 0.86 & 0.61 & 1.16 & C2 & $1.34^{*}$ & B2 & $1.34^{*}$ \\
13 & 3 & 3 & 0.85 & 0.61 & 1.15 & C3 & 1.34 & B3 & $1.18^{*}$ \\
12 & 3 & 3 & 0.85 & 0.60 & 1.16 & & & & \\
11 & 3 & 3 & 0.85 & 0.58 & 1.17 & & & &
\end{tabular}

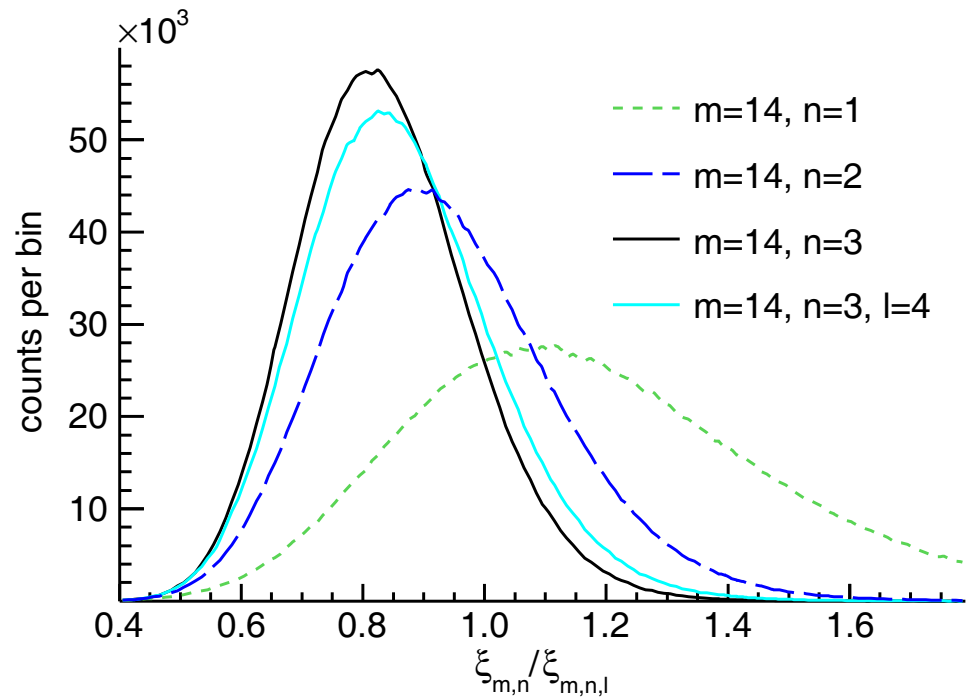

Figure 3: Simulated distributions of $\xi_{m, n, l}$ for various values of $m, n$ and $l$. 
consider the case when $l$ out of $m$ chains have only two decays, while the rest are "complete" $\left(n_{i}=n=3\right)$ with three decays. In fig. 3 the distribution of $\xi_{m, n, l}$ is shown for $m=14, n=3$, and $l=4$. Increasing $l$ from zero to $m$, the distribution gradually changes from the respective distributions from $\xi_{m, n=3}$ to $\xi_{m, n=2}$. Some relevant $90 \%$ confidence intervals are presented in the left part of table 1 .

For the complete set of $m=14$ short chains the value of $\xi_{m, n_{i}}$ is 1.30 - well above the corresponding $90 \%$ confidence interval $[0.62,1.15]$ obtained for $\xi_{14,3,4}$. Therefore, it is very unlikely that it is a mistake to conclude that not all chains originate from the same radioactive species. To investigate the data set more carefully, one chain at the time was excluded from the full set. The resulting values of $\xi_{m, n_{i}}$ are given in the first column in table 1 behind the respective excluded chain. When interpreting the values, attention must be paid to the fact that $l$ changes when a chain with only two decays is excluded. The values marked with * should thus be compared with the interval $[0.61,1.15]\left(\xi_{13,3,3}\right)$ and the others with $[0.61,1.16]\left(\xi_{13,3,4}\right)$. The only way to reach the $90 \%$ confidence interval by excluding only one chain is to exclude chain D3.

Excluding chains B3 or $\mathrm{C} 4$ also diminishes $\xi_{m, n_{i}}$ somewhat, which draws the attention to those chains. If chains $\mathrm{C} 4$ and $\mathrm{B} 3$ are both excluded, the twelve remaining chains lead to $\xi_{m, n_{i}}=1.06$. This is within the $90 \%$ confidence interval $[0.60,1.16]$ for $\xi_{12,3,3}$. Hence the exclusion of these two chains pose an alternative to excluding D3.

When excluding all three chains D3, C4 and B3 the value becomes $\xi_{m, n_{i}}$ $=0.71$. This is below the expectation value 0.85 . Since it is within the corresponding interval $[0.58,1.17]$, there is no strong argument not to exclude all of them. However, since smaller changes to the data set are sufficient to account for the $90 \%$ confidence interval, such trimming of the data cannot be motivated by only an analysis of the spread of correlation times.

The conclusion from the statistical assessment is that the short chains most likely do not come from the same radioactive species. There are several different subsets of the data that fulfill the requirement of a $\xi_{m, n_{i}}$ within the $90 \%$ confidence interval. This test cannot unambigously tell how the data set should be divided, but it shows that separation into different species is necessary. A proper inclusion of decay energies in the statistical analysis should be considered as well. 


\section{Summary}

Modern spectroscopy provide first glimpses of solid experimental information for detailed nuclear structure assessments of the heaviest atomic nuclei available today. Furthermore, the increasing number of decay chains associated with element 115 allows extended assessments of the data, which is relevant for assigning a given decay chain to a certain isotope (or isomer of an isotope). Such studies lead to possible EC or SF branches of otherwise long element 115 decay chains and/or parallel decay sequences starting from the same isotope. Ultimately, all these features have to find their explanation in the underlying nuclear structure of the heaviest elements.

\section{Acknowledgements}

NN2015 attendance and these Proceedings are supported by the Swedish Research Council and the Royal Physiographic Society in Lund. The assembly of TASISpec would have been impossible without generous grants from the Royal Physiographic Society in Lund and support from the Euroball Owners Committee. The GSI experiment on element 115 was supported by the European Community FP7 - Capacities ENSAR No. 262010.

\section{References}

[1] M. Schädel, Phil. Trans. R. Soc. A 373, 20140191 (2015).

[2] S.G. Nilsson et al., Nucl. Phys. A131, 1 (1969).

[3] M. Bender, W. Nazarewicz, and P.-G. Reinhard, Phys. Lett. B515, 42 (2001).

[4] S. Ćwiok, P.H. Heenen, and W. Nazarewicz, Nature 433, 705 (2005).

[5] J. Dvorak et al., Phys. Rev. Lett. 97, 242501 (2006).

[6] R.-D. Herzberg and P.T. Greenlees, Prog. Part. Nucl. Phys. 61, 674 (2008).

[7] Y.T. Oganessian, J. Phys. G 34, R165 (2007); Radiochim. Acta 99, 429 (2011).

[8] Y.T. Oganessian et al., Phys. Rev. C 87, 054621 (2013).

[9] R.C. Barber et al., Pure Appl. Chem. 83(7), 1485 (2011).

[10] G.T. Seaborg and W.D. Loveland, The Elements Beyond Uranium (WileyInterscience, New York, 1990).

[11] C.E. Bemis, Jr. et al., Phys. Rev. Lett. 31, 647 (1973).

[12] T.A. Carlsson et al., Nucl. Phys. A135, 57 (1969); At. Data Nucl. Data Tables 19, 153 (1977).

[13] H.G.J. Moseley, Phil. Mag. 26, 1024 (1913).

[14] D. Rudolph et al., Phys. Rev. Lett. 111, 112502 (2013).

[15] D. Rudolph, L.G. Sarmiento and U. Forsberg, submitted to AIP Conf. Proc. 
[16] Ch.E. Düllmann, Proc. $5^{\text {th }}$ International Conference on Fission and Properties of Neutron-Rich Nuclei, 2012, Sanibel Island, Florida, Eds. J.H. Hamilton and A.V. Ramayya, World Scientific, Singapore (2013), p. 271.

[17] D. Rudolph et al., JPS Conf. Proc. 6, 010026 (2015).

[18] J. Khuyagbaatar et al., Phys. Rev. Lett. 112, 172501 (2014).

[19] A. Yakushev et al., Inorg. Chem. 53, 1624 (2014).

[20] Yu. Ts. Oganessian et al., Phys. Rev. C 69, 021601(R) (2004).

[21] Yu. Ts. Oganessian et al., Phys. Rev. C 87, 014302 (2013).

[22] U. Forsberg et al., submitted to Nucl. Phys. A; http://arxiv.org/abs/1502.03030.

[23] J.M. Gates et al., Phys. Rev. C 92, 021301(R) (2015).

[24] F.P. Heßberger et al., Nucl. Instrum. Meth. A 274, 522 (1989).

[25] K.-H. Schmidt, Eur. Phys. J. A 8, 141 (2000).

[26] D. Rudolph et al., Acta Phys. Pol. B45, 263 (2014). 